\title{
Early Gamma Knife stereotactic radiosurgery to the tumor bed of resected brain metastasis for improved local control
}

\author{
Clinical article
}

\author{
Christian Iorio-Morin, M.D., Ph.D., ${ }^{1,3}$ Laurence Masson-Côté, M.D., F.R.C.P.C., ${ }^{2-4}$ \\ Youssef Ezahr, M.D., ${ }^{1,3}$ Jocelyn BlanchaRd, M.D., F.R.C.S.C., ${ }^{1,3}$ \\ AnNie Ebacher, M.D., F.R.C.P.C., ${ }^{2,3}$ ANd DaVId Mathieu, M.D., F.R.C.S.C. ${ }^{1,3,4}$ \\ ${ }^{1}$ Division of Neurosurgery, Department of Surgery, and ${ }^{2}$ Division of Radiation Oncology, Department of \\ Nuclear Medicine and Radiobiology, Faculté de médecine et des sciences de la santé, Université de \\ Sherbrooke; ${ }^{3}$ Centre Hospitalier Universitaire de Sherbrooke; and ${ }^{4}$ Centre de Recherche du Centre \\ Hospitalier Universitaire de Sherbrooke, Sherbrooke, Québec, Canada
}

\begin{abstract}
Object. Optimal case management after surgical removal of brain metastasis remains controversial. Although postoperative whole-brain radiation therapy (WBRT) has been shown to prevent local recurrence and decrease deaths, this modality can substantially decrease neurocognitive function and quality of life. Stereotactic radiosurgery (SRS) can theoretically achieve the same level of local control with fewer side effects, although studies conclusively demonstrating such outcomes are lacking. To assess the effectiveness and safety profile of tumor bed SRS after resection of brain metastasis, the authors performed a retrospective analysis of 110 patients who had received such treatment at the Centre Hospitalier Universitaire de Sherbrooke. They designed the study to identify risk factors for local recurrence and placed special emphasis on factors that could potentially be addressed.

Methods. Patients who had received treatment from 2004 through 2013 were included if they had undergone surgical removal of 1 or more brain metastases and if the tumor bed was treated by SRS regardless of the extent of resection or prior WBRT. All cases were retrospectively analyzed for patient and tumor-specific factors, treatment protocol, adverse outcomes, cavity outcomes, and survival for as long as follow-up was available. Univariate and multivariate Cox regression analyses were performed to identify risk factors for local recurrence and predictors of increased survival times.

Results. Median patient age at first SRS treatment was 58 years (range 37-84 years). The most frequently diagnosed primary tumor was non-small cell lung cancer. The rate of gross-total resection was $81 \%$. The median Karnofsky Performance Scale score was $90 \%$. Tumor bed SRS was performed at a median of 3 weeks after surgery. Median follow-up and survival times were 10 and 11 months, respectively. Actuarial local control of the cavity at 12 months was $73 \%$; median time to recurrence was 6 months. According to multivariate analysis, risk factors for recurrence were a longer surgery-to-SRS delay $(\mathrm{HR} 1.625, \mathrm{p}=0.003)$ and a lower maximum radiation dose delivered to the cavity (HR 0.817, $\mathrm{p}=0.006$ ). Factors not associated with increased recurrence were subtotal or piecemeal resections, prior WBRT, histology of the primary tumor, and larger cavity volume. No factors predictive of survival were identified. Symptomatic radiation-induced enhancement occurred in $6 \%$ of patients and leptomeningeal dissemination in $11 \%$. Pathologically confirmed radiation-induced necrosis occurred in $1(0.9 \%)$ patient.

Conclusions. Adjuvant tumor bed SRS after the resection of brain metastasis is a valuable alternative to adjuvant WBRT. Risk factors for local recurrence are lower maximum radiation dose and a surgery-to-SRS delay longer than 3 weeks. Outcomes were not worse for patients who had undergone prior WBRT and subtotal or piecemeal resections. Pending the results of prospective randomized controlled trials, the authors' study supports the safety and efficacy of adjuvant SRS after resection of brain metastasis. SRS should be performed as early as possible, ideally within 3 weeks of the surgery.
\end{abstract}

(http://thejns.org/doi/abs/10.3171/2014.7.GKS141488)

\section{Key Words • Gamma Knife • tumor bed • brain metastasis • stereotactic radiosurgery}

RAIN metastases are a significant source of illness and death among cancer patients ${ }^{8}$ and will eventually develop in about $20 \%-40 \%$ of patients with

Abbreviations used in this paper: $\mathrm{SRS}=$ stereotactic radiosurgery; $\mathrm{WBRT}=$ whole-brain radiation therapy. a primary extracranial neoplasm. ${ }^{9}$ The intent of current optimal case management remains mostly palliative. Resection has been shown to improve neurological symptoms, improve functional independence, and increase survival times. ${ }^{19}$ However, surgery alone is associated with a $46 \%-59 \%$ rate of local recurrence, presumably because 
any residual microscopic disease is left unaddressed. ${ }^{4} \mathrm{Ad}-$ juvant whole-brain radiation therapy (WBRT) can treat both local and distant microscopic disease and has been shown to decrease local recurrence in the first year by $10 \%-28 \%$. WBRT also decreases the incidence of new distant brain metastasis from $37 \%$ to $14 \%$ and decreases neurological deaths without, however, increasing overall survival times. ${ }^{13,18}$ Yet WBRT is not without consequences. A recent study has shown that in addition to physical symptoms such as hair loss and skin reactions, WBRT induces neurocognitive decline; symptoms are evident as early as 4 months after treatment. ${ }^{5}$ Because neurocognitive function is known to be correlated with quality of life,$^{14}$ this finding is highly relevant clinically. In theory, the same level of local control - with fewer side effects could be achieved by performing stereotactic radiosurgery (SRS) to the tumor bed after resection.

To assess the effectiveness and safety of this approach, we performed a retrospective analysis of 110 patients who had received such treatment at our center. We designed the study to identify risk factors for local recurrence and placed special emphasis on factors that could potentially be addressed to improve treatment parameters and, ultimately, patient outcomes. Our institutional review board approved this study.

\section{Methods}

All patients treated at our center (Centre Hospitalier Universitaire de Sherbrooke Gamma Knife center) during 2004-2013 were screened for eligibility. Patients were included in the series if they had undergone Gamma Knife SRS to the tumor bed of pathologically confirmed and surgically removed brain metastasis. The initial database query yielded 133 eligible patients. After patient chart reviews, 23 patients were excluded: 4 did not meet the inclusion criteria, 11 had no available follow-up information, and 8 had received treatment more than 2 months after the surgery, having been referred to our center for already ongoing local recurrence. The final series therefore included 110 patients and 113 cavities.

For each patient, study variables were collected by using Excel for Mac 2011, version 14.4.2 (Microsoft Corp.). Statistical analyses were performed by using SPSS Statistics, version 22.0.0.0 (IBM). Statistical significance was defined as $\mathrm{p}<0.05$. A primary analysis involved the complete series. Secondary subgroup analyses involved only patients who had undergone gross-total resection and/ or without prior WBRT and yielded identical results to the analysis of the full series (data not shown). Analyzed outcomes were local control (defined as the absence of confirmed tumor growth within or adjacent to the postoperative cavity on follow-up MR images) and survival times. Conditional forward stepwise Cox regression analyses were performed to identify risk factors for outcomes in univariate and multivariate models. The multivariate analysis was initially performed by using only the variables for which $\mathrm{p}$ was $<0.15$ in the univariate analysis (data not shown). Additional variables were then added to the regression until all the variables of the univariate model were included with no effect on the statistical significance of identified risk factors. Further actuarial analyses were performed by using the Kaplan-Meier estimator. For local control analysis, cases were censored at the last available imaging study. Different KaplanMeier distributions were compared by using the log-rank (Mantel-Cox) test. Continuous variables were compared by using the Pearson correlation coefficient. All times reported are relative to the date of the SRS. Final figures were produced by using IBM SPSS Statistics and Prism 6.0 (GraphPad Software Inc.).

\section{Patient Population}

Patient characteristics are presented in Table 1. The female/male ratio was 1.33:1, and median patient age was 58 years. For most patients, functional status was good at the time of SRS; the median Karnofsky Performance Scale score was 90\%, median Radiation Therapy Oncology Group RPA (recursive partitioning analysis) class was II, and $62 \%$ had no focal neurological deficit. The most frequent primary pathology was non-small cell lung cancer, followed by breast and colorectal cancers. For $40 \%$ of patients, the brain metastasis was diagnosed at the same time as the primary cancer. For the other patients, brain metastasis was diagnosed a median of 24 months after diagnosis of the primary cancer.

Surgical resection of the metastasis was performed at our center for 78 (71\%) patients; the others were referred specifically for SRS after having undergone surgery at other institutions. Metastases were removed en bloc for $42 \%$ of patients at a median of 9 days after the diagnosis of brain involvement. MR image-confirmed gross-total resection was achieved for $81 \%$ of cases.

\section{Radiosurgery Procedures}

All SRS procedures were performed by using the parameters detailed in Table 2 . In brief, after induction of conscious sedation and local anesthesia, a stereotactic Leksell $\mathrm{G}$ frame (Elekta AB) was attached to the patient's head. Volumetric T1-weighted images with and without contrast enhancement were obtained and used to define the target. The neurosurgeon, radiation oncologist, and medical physicist devised a treatment plan by using Leksell GammaPlan software (Elekta AB). The 50\% isodose line was used for prescription in most cases, and the median margin and maximum doses delivered to the tumor bed were 18 and 36 Gy, respectively. The prescription isodose line included an approximate margin of $1 \mathrm{~mm}$ of brain tissue around the resection edge and covered any residual enhancement or tumor in cases of partial resection. Any unresected remote brain metastasis was also irradiated during the procedure. After treatment, patients were briefly observed and discharged to home the same day.

Clinical and radiological follow-up examinations were performed 2 months after SRS and then every 3 months.

\section{Results}

The median follow-up time was 10 months (range 1.4-84.2 months). At the time of analysis, $75 \%$ of cavi- 
TABLE 1: Patient characteristics

\begin{tabular}{|c|c|}
\hline Characteristic & Value \\
\hline no. of patients & 110 \\
\hline no. of cavities & 113 \\
\hline sex, no. (\%) of patients & \\
\hline $\mathrm{F}$ & $63(57)$ \\
\hline M & $47(43)$ \\
\hline median age in yrs at SRS (range) & $58(37-84)$ \\
\hline $\begin{array}{l}\text { neurological status at SRS, no. (\%) of cavities } \\
\text { no deficit } \\
\text { focal deficit }\end{array}$ & $\begin{array}{l}70(62) \\
43(38)\end{array}$ \\
\hline median (range) KPS score at SRS & $90(50-100)$ \\
\hline $\begin{array}{l}\text { RTOG RPA class at SRS, no. (\%) of cavities } \\
\text { I } \\
\text { III }\end{array}$ & $\begin{array}{l}34(30) \\
70(62) \\
9(8)\end{array}$ \\
\hline $\begin{array}{l}\text { primary pathology, no. (\%) of cavities } \\
\text { NSCLC } \\
\text { breast } \\
\text { colorectal } \\
\text { melanoma } \\
\text { renal } \\
\text { others }\end{array}$ & $\begin{array}{c}57(50) \\
15(13) \\
14(12) \\
11(10) \\
5(5) \\
11(10)\end{array}$ \\
\hline $\begin{array}{l}\text { time of } 1 \text { st brain metastasis after primary diagnosis } \\
\text { synchronous diagnosis, no. (\%) of patients } \\
\text { median time (range) for nonsynchronous diagnosis, } \\
\text { mos }\end{array}$ & $\begin{array}{l}44(40) \\
24(0.1-188)\end{array}$ \\
\hline prior WBRT, no. (\%) of cavities & $17(15)$ \\
\hline median (range) delay btwn WBRT \& SRS, mos & $7(0-96)$ \\
\hline $\begin{array}{l}\text { median (range) delay btwn brain metastasis \& surgery, } \\
\text { days }\end{array}$ & $9(0-2908)$ \\
\hline $\begin{array}{l}\text { resection, no. (\%) of cavities } \\
\text { en bloc } \\
\text { piecemeal }\end{array}$ & $\begin{array}{l}33(42) \\
45(58)\end{array}$ \\
\hline $\begin{array}{l}\text { extent of resection, no. (\%) of cavities } \\
\text { gross total } \\
\text { subtotal }\end{array}$ & $\begin{array}{l}92(81) \\
21(19)\end{array}$ \\
\hline median (range) delay btwn brain surgery \& SRS, wks & $3(0.3-7.6)$ \\
\hline $\begin{array}{l}\text { median (range) no. of treated metastases (including } \\
\text { cavity) at 1st SRS }\end{array}$ & $1(1-9)$ \\
\hline
\end{tabular}

* KPS = Karnofsky Performance Scale; NSCLC = non-small cell lung cancer; RTOG RPA = Radiation Therapy Oncology Group recursive partitioning analysis.

ties were free of recurrence. Actuarial analysis (Table 3) estimated local control at $6,12,24$, and 36 months to be $84 \%, 73 \%, 64 \%$, and $58 \%$, respectively. Recurrence was observed at a median of 6 months (range 1-57 months) after SRS. According to univariate and multivariate analyses, risk factors for recurrence were a longer surgeryto-SRS delay (HR 1.625, 95\% CI 1.183-2.231, $\mathrm{p}=0.003$ ) and a lower maximum radiation dose delivered to the cavity (HR 0.817, 95\% CI 0.708-0.943, p = 0.006) (Table 4). When stratified by the surgery-to-SRS delay, increased delays were associated with decreased rates of 12-month actuarial local control (Fig. 1). According to Kaplan-Mei-
TABLE 2: SRS treatment parameters

\begin{tabular}{llcr}
\hline \multicolumn{1}{c}{ Parameter } & Mean & Median & Range \\
\hline margin dose (Gy) & 17.1 & 18 & $10-20$ \\
maximum dose (Gy) & 35 & 36 & $20-53$ \\
isodose line (\%) & 49.1 & 50 & $30-80$ \\
isocenters (no.) & 16.7 & 15 & $1-49$ \\
treatment vol (cm $\left.{ }^{3}\right)$ & 13 & 12 & $0.6-43$ \\
\hline
\end{tabular}

er analyses, the cutoff (maximum delay) most predictive of local control was 3 weeks or sooner (Fig. 2) $(p=0.003)$, although using a 4-week cutoff still identified statistically different distributions ( $\mathrm{p}=0.016$, data not shown). The surgery-to-SRS delay was inversely proportional to the size of the cavity $(\mathrm{p}=0.019)$ and directly proportional to the margin dose $(\mathrm{p}=0.025)$. With regard to treatment parameters, maximum dose directly correlated with margin dose $(\mathrm{p}<0.01)$ and was inversely proportional to the cavity volume $(\mathrm{p}<0.01)$, although the effect of the 2 latter factors on recurrence was not statistically significant. All other tested factors were also not significantly associated with increased risk for recurrence and included subtotal or piecemeal resections, prior WBRT, and primary tumor pathology. For primary tumor pathology, the effect of radioresistant (melanoma, renal cell carcinoma) versus nonradioresistant (all others) tumors was specifically tested and yielded no difference in outcomes.

The median survival time was 11 months (range 1.4-84 months); $44 \%$ of patients were still alive at the time of analysis. Actuarial survival rate data are presented in Table 3. The cause of death was known to be neurological (i.e., related to the progression of the cerebral disease, including systemic complications of neurological symptoms) in only $23 \%$ of patients; most others died of a systemic condition. No risk factor shown in Table 4 was significantly associated with decreased survival time according to the multivariate model, although progression of the primary tumor during the follow-up period showed a statistically significant trend toward decreased survival time according to the univariate analysis ( $\mathrm{p}=0.045$, data not shown).

Radiation-induced T2 anomaly and enhancement occurred in $19 \%$ and $32 \%$ of cavities, respectively. Enhancement resolved spontaneously during the follow-up period for 8 cavities, suggesting that the real incidence of permanent radiation-induced changes in our series was $22 \%$ at most. Necrosis was pathologically proven for only 1 patient, who underwent surgery because symptomatic recurrence was considered the most likely diagnosis before pathological analysis had confirmed the necrosis. Radiation-induced enhancement of the cavity was symptomatic in only $7(6 \%)$ patients. Leptomeningeal dissemination was seen in $11 \%$ of patients and was not associated with any particular primary pathology. Subsequent distant brain metastasis occurred in $54 \%$ of patients. Subsequent WBRT, SRS, and surgery were eventually performed in $28 \%, 38 \%$, and $12 \%$ of patients, respectively, and mostly for the management of other metastases not associated with the cavity. Subsequent WBRT, SRS, and surgery 
TABLE 3: Actuarial control and survival

\begin{tabular}{ccccc}
\hline & \multicolumn{3}{c}{$\%$ Control } \\
\cline { 2 - 5 } Variable & 6 Mos & 12 Mos & 24 Mos & 36 Mos \\
\hline actuarial local control (cavity) & & & 64 & 58 \\
all patients & 84 & 73 & 87 & 72 \\
surgery-SRS delay $\leq 3$ wks & 94 & 87 & 46 & 46 \\
surgery-SRS delay >3 wks & 77 & 61 & 38 & 26 \\
actuarial survival & 83 & 63 & & \\
\hline
\end{tabular}

were performed at a median of 5, 6, and 11 months, respectively, after the initial SRS.

\section{Discussion}

During the past few years, tumor bed SRS has been increasingly used as an alternative to WBRT after brain metastasis resection. Several other retrospective studies have supported the safety and efficacy of adjuvant SRS in this setting. ${ }^{3,7,16}$ Our rate of crude local control (75\%) is within the reported range of 70\%-100\% and near the 79\% average,$^{20}$ as are our other outcomes of an 11-month median survival time (reported range $10-20$ months), $1 \%$ rate of operated radiation-induced necrosis (published rate of $3 \%$ ), ${ }^{12}$ and $11 \%$ rate of meningeal dissemination (published rate of $13 \%) .{ }^{2}$ However, our series is larger and provides

\section{TABLE 4: Risk factors for local recurrence*}

\begin{tabular}{|c|c|c|}
\hline \multirow[b]{2}{*}{ Risk Factor } & \multicolumn{2}{|c|}{$p$ Value } \\
\hline & $\begin{array}{c}\text { Univariate } \\
\text { Analysis }\end{array}$ & $\begin{array}{c}\text { Multivariate } \\
\text { Analysis }\end{array}$ \\
\hline \multicolumn{3}{|l|}{ patient characteristic } \\
\hline female sex & 0.775 & 0.722 \\
\hline age & 0.648 & 0.908 \\
\hline subtotal resection & 0.578 & 0.823 \\
\hline piecemeal resection & 0.912 & 0.803 \\
\hline prior WBRT & 0.596 & 0.745 \\
\hline focal neurological deficit at SRS & 0.260 & 0.809 \\
\hline KPS score at SRS & 0.240 & 0.392 \\
\hline RTOG RPA class at SRS & 0.240 & 0.141 \\
\hline \multicolumn{3}{|l|}{ tumor characteristic } \\
\hline primary pathology & 0.504 & 0.187 \\
\hline prior extracranial radiotherapy & 0.884 & 0.544 \\
\hline progression of primary & 0.094 & 0.551 \\
\hline cavity vol & 0.544 & 0.770 \\
\hline cavity location & 0.585 & 0.618 \\
\hline \multicolumn{3}{|l|}{ SRS characteristic } \\
\hline surgery-to-SRS delay & 0.024 & 0.003 \\
\hline margin dose $\leq 16$ Gy & 0.106 & 0.152 \\
\hline isodose line & 0.397 & 0.555 \\
\hline maximum dose & 0.035 & 0.006 \\
\hline no. of isocenters & 0.968 & 0.447 \\
\hline
\end{tabular}

longer follow-up times than do most published reports of other studies, ${ }^{7}$ thereby reducing the positive control and survival biases associated with short-term or nonreported follow-ups. These coherent results suggest that our study population is probably similar to the cohorts in other published studies and that conclusions from our risk factor analysis might be applicable to those patients as well.

Previously identified risk factors for local recurrence include subtotal resection, larger treatment volume, and lower margin dose. ${ }^{7,16}$ In our series, 2 factors were significantly associated with higher rates of treatment failure: a lower maximum dose of radiation and a longer delay between resection and SRS.

\section{Radiation Dose}

Multiple authors have identified use of a low margin dose as a risk factor for local recurrence. ${ }^{10,16,22}$ In our series, we found a trend toward better local control when higher margin doses were used, but the trend did not meet statistical significance in any analysis (we tested the effect of both the continuous variable and a binary variable with a cutoff of $\leq 16 \mathrm{~Gy}$ in both the univariate and multivariate models). There was, however, in both models a significant association between higher maximum dose and better local control. Furthermore, maximum dose correlated with margin dose and was inversely proportional to the cavity volume. It might be that the wider range of possible maxi-

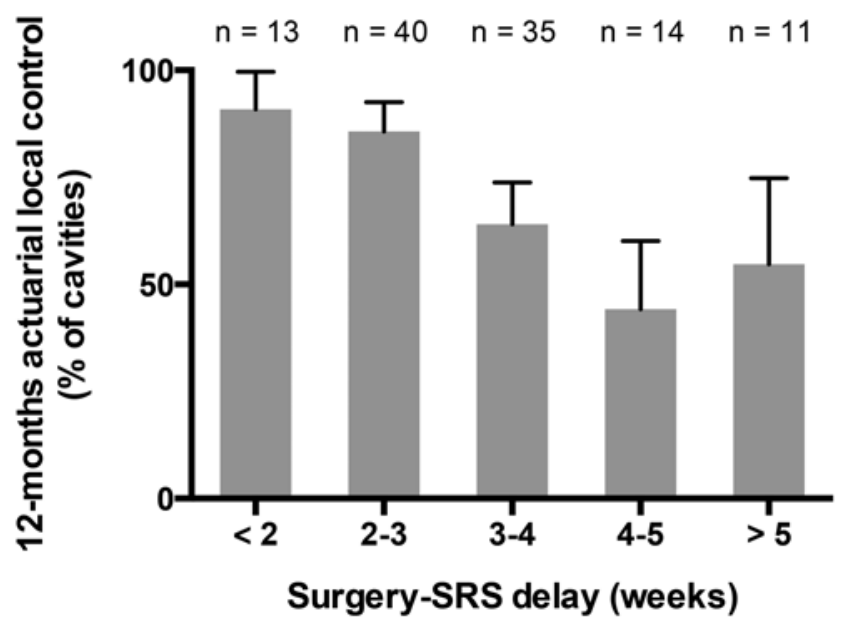

Fig. 1. Actuarial local control rates at 12 months after SRS, stratified by the surgery-to-SRS delay. Error bars represent standard deviations. The numbers above the histogram represent the number of cavities in each group (total $=113$ cavities). 


\section{Early postoperative tumor bed SRS for resected brain metastasis}

mum doses (20-53 Gy) in our treatment plans compared with margin doses (10-20 Gy) made statistical significance easier to reach for the former variable. Although it is possible that the effect of the maximum dose on local control might be a statistical error in itself, an error is unlikely given the internal consistency of our data (between maximum dose, margin dose, and cavity volume) and their agreement with that of other authors. ${ }^{10,16,22}$ Until future studies further clarify this issue, our data support the notion that a higher dose of radiation, marginal or maximal, improves local control.

Surgery-to-SRS Delay

Our study demonstrated an effect of the surgery-toSRS delay on local recurrence. Current practice at multiple institutions, including ours, is to perform SRS within 6 weeks after the resection..$^{12,17}$ The rationale for such a delay is 3-fold: 1) time enables the patient to recover from the surgery before the SRS; 2) few metastases have already recurred after 6 weeks if gross-total resection has been achieved; and 3) time allows for cavity shrinkage, thereby reducing the treatment volume and enabling use of a higher margin dose. Although the latter assumption has recently been challenged,${ }^{11}$ in our series, the surgeryto-SRS delay was indeed inversely proportional to the size of the cavity and directly proportional to the margin dose. However, regardless of the benefits on treatment parameters, a longer surgery-to-SRS delay clearly correlated with increased local recurrence (Fig. 1). A cutoff of 3 weeks or sooner (Fig. 2) best stratified patients in Kaplan-Meier analyses. The delay substantially affected actuarial local control rates; estimated 6-month control rates dropped from $94 \%$ to $77 \%$ if SRS was performed more than 3 weeks after resection (Table 3). A longer surgery-to-SRS delay might lead to an increased spread of microscopic disease that is harder to target because it is not yet enhancing. To the best of our knowledge, only 2 other studies reported their surgery-to-SRS delay, ${ }^{7,12,17}$ and ours is the first to demonstrate the effect. Perhaps the most useful aspect of this finding is that, unlike most other treatment parameters, this delay can actually be addressed quite safely and easily.

\section{Prior Radiation Exposure}

In our series, prior exposure of the neoplastic cells to radiation-either through irradiation of the primary cancer or through prior WBRT-was not associated with increased local recurrence. This finding suggests that any acquired radioresistance might not be clinically relevant with regard to tumor bed SRS sensitivity. Prior WBRT was also not associated with increased SRS-induced T2 anomalies or enhancement (data not shown); it is noteworthy that in our series, the only patient who underwent surgery for radiation-induced necrosis had not undergone WBRT before SRS. Together, these observations support both the safety and relevance of tumor bed SRS for patients previously treated with WBRT.

\section{Extent of Resection}

Subtotal resections are often excluded from cavity-

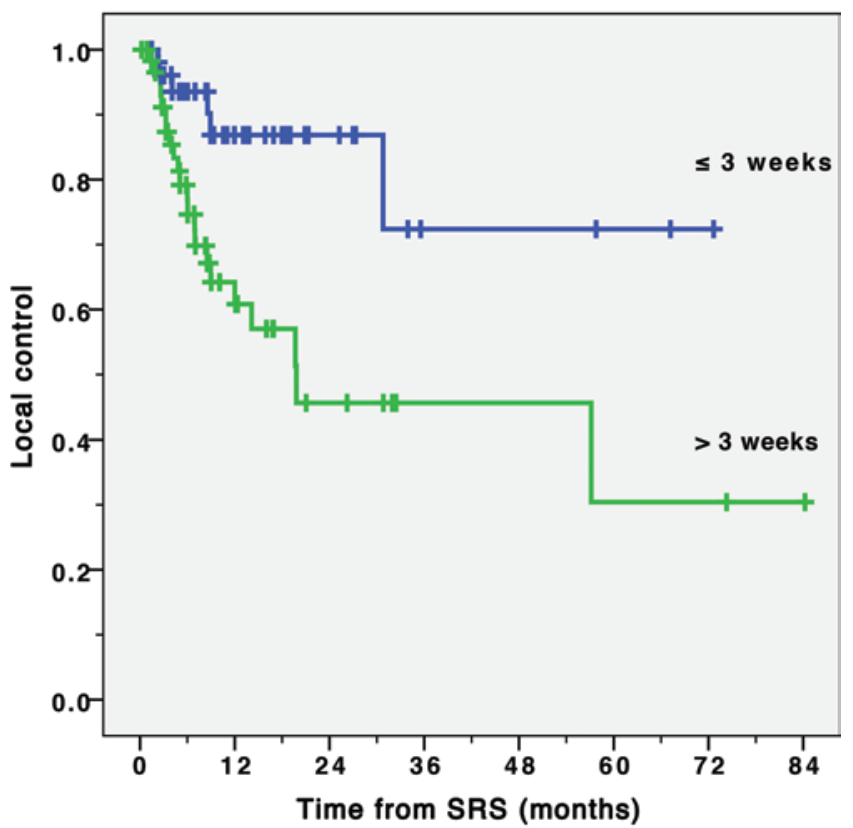

Fig. 2. Actuarial analysis of local control stratified by the surgery-toSRS delay (log-rank, $p=0.003$ ).

SRS series ${ }^{16,21}$ and in a recent meta-analysis of 629 patients were found to be associated with decreased local control. ${ }^{7}$ However, in the largest cohort in which these patients were included, subtotal resection was not associated with increased local recurrence. ${ }^{12}$ Likewise, in our series, neither subtotal nor piecemeal resection was associated with increased recurrence. Indeed, the effectiveness of SRS as the primary treatment for brain metastasis is well established., ${ }^{1,6,15}$ Our results further suggest that treatment parameters used for tumor bed SRS can control macroscopic residual disease as well as local microscopic contaminations, as long as these are included in the treatment plan. This observation is of particular relevance with regard to metastases located near eloquent areas, where pushing the resection too far might compromise function. Although our data do not enable us to specify up to which residual volume this observation holds true, they support the idea that when gross-total resection is impossible, an effective strategy might be staged surgical debulking followed by cavity plus residual SRS.

\section{Conclusions}

Adjuvant tumor bed SRS after resection of brain metastasis is a valuable alternative to adjuvant WBRT. In our series, local control rates provided by SRS compared favorably with those published for WBRT. Lower maximum radiation dose and a surgery-to-SRS delay longer than 3 weeks were risk factors for local recurrence. Outcomes were not worse for patients who had previously undergone WBRT and subtotal or piecemeal resections. Pending the results of prospective randomized controlled trials, our study supports the safety and efficacy of adjuvant SRS after resection of brain metastasis. We encourage clinicians to perform the SRS procedure as early as possible, ideally within 3 weeks of the surgery. 


\section{Acknowledgment}

We thank Modou Sene for assistance with statistical analyses.

\section{Disclosure}

The authors report no conflict of interest concerning the materials or methods used in this study or the findings specified in this paper. Dr. Iorio-Morin receives an M.D.-Ph.D. scholarship from the Fonds de recherche du Québec-Santé.

Author contributions to the study and manuscript preparation include the following. Conception and design: Mathieu, Iorio-Morin. Acquisition of data: Iorio-Morin, Ezahr. Analysis and interpretation of data: Mathieu, Iorio-Morin, Masson-Côté. Drafting the article: Iorio-Morin. Critically revising the article: Mathieu, Masson-Côté. Reviewed submitted version of manuscript: Mathieu, Masson-Côté. Approved the final version of the manuscript on behalf of all authors: Mathieu. Statistical analysis: Iorio-Morin. Administrative/technical/ material support: Blanchard, Ebacher. Study supervision: Mathieu.

\section{References}

1. Aoyama H, Shirato H, Tago M, Nakagawa K, Toyoda T, Hatano K, et al: Stereotactic radiosurgery plus whole-brain radiation therapy vs stereotactic radiosurgery alone for treatment of brain metastases: a randomized controlled trial. JAMA 295:2483-2491, 2006

2. Atalar B, Modlin LA, Choi CYH, Adler JR, Gibbs IC, Chang $\mathrm{SD}$, et al: Risk of leptomeningeal disease in patients treated with stereotactic radiosurgery targeting the postoperative resection cavity for brain metastases. Int J Radiat Oncol Biol Phys 87:713-718, 2013

3. Baschnagel AM, Meyer KD, Chen PY, Krauss DJ, Olson RE, Pieper DR, et al: Tumor volume as a predictor of survival and local control in patients with brain metastases treated with Gamma Knife surgery. Clinical article. J Neurosurg 119: 1139-1144, 2013

4. Baumert BG, Rutten I, Dehing-Oberije C, Twijnstra A, Dirx MJM, Debougnoux-Huppertz RMTL, et al: A pathologybased substrate for target definition in radiosurgery of brain metastases. Int J Radiat Oncol Biol Phys 66:187-194, 2006

5. Chang EL, Wefel JS, Hess KR, Allen PK, Lang FF, Kornguth DG, et al: Neurocognition in patients with brain metastases treated with radiosurgery or radiosurgery plus whole-brain irradiation: a randomised controlled trial. Lancet Oncol 10: 1037-1044, 2009

6. Elaimy AL, Mackay AR, Lamoreaux WT, Fairbanks RK, Demakas JJ, Cooke BS, et al: Clinical outcomes of stereotactic radiosurgery in the treatment of patients with metastatic brain tumors. World Neurosurg 75:673-683, 2011

7. Gans JH, Raper DMS, Shah AH, Bregy A, Heros D, Lally BE, et al: The role of radiosurgery to the tumor bed after resection of brain metastases. Neurosurgery 72:317-326, 2013

8. Gaspar L, Scott C, Rotman M, Asbell S, Phillips T, Wasserman T, et al: Recursive Partitioning Analysis (RPA) of prognostic factors in three Radiation Therapy Oncology Group (RTOG) brain metastases trials. Int J Radiat Oncol Biol Phys 37:745751,1997

9. Gavrilovic IT, Posner JB: Brain metastases: epidemiology and pathophysiology. J Neurooncol 75:5-14, 2005

10. Iwai Y, Yamanaka K, Yasui T: Boost radiosurgery for treatment of brain metastases after surgical resections. Surg Neurol 69:181-186, 2008
11. Jarvis LA, Simmons NE, Bellerive M, Erkmen K, Eskey CJ, Gladstone DJ, et al: Tumor bed dynamics after surgical resection of brain metastases: implications for postoperative radiosurgery. Int J Radiat Oncol Biol Phys 84:943-948, 2012

12. Jensen CA, Chan MD, McCoy TP, Bourland JD, deGuzman $\mathrm{AF}$, Ellis TL, et al: Cavity-directed radiosurgery as adjuvant therapy after resection of a brain metastasis. Clinical article. $\mathbf{J}$ Neurosurg 114:1585-1591, 2011

13. Kocher M, Soffietti R, Abacioglu U, Villà S, Fauchon F, Baumert BG, et al: Adjuvant whole-brain radiotherapy versus observation after radiosurgery or surgical resection of one to three cerebral metastases: results of the EORTC 22952-26001 study. J Clin Oncol 29:134-141, 2011

14. Li J, Bentzen SM, Li J, Renschler M, Mehta MP: Relationship between neurocognitive function and quality of life after whole-brain radiotherapy in patients with brain metastasis. Int J Radiat Oncol Biol Phys 71:64-70, 2008

15. Linskey ME, Andrews DW, Asher AL, Burri SH, Kondziolka $\mathrm{D}$, Robinson PD, et al: The role of stereotactic radiosurgery in the management of patients with newly diagnosed brain metastases: a systematic review and evidence-based clinical practice guideline. J Neurooncol 96:45-68, 2010

16. Luther N, Kondziolka D, Kano H, Mousavi SH, Engh JA, Niranjan A, et al: Predicting tumor control after resection bed radiosurgery of brain metastases. Neurosurgery 73:1001-1006, 2013

17. Mathieu D, Kondziolka D, Flickinger JC, Fortin D, Kenny B, Michaud K, et al: Tumor bed radiosurgery after resection of cerebral metastases. Neurosurgery 62:817-824, 2008

18. Patchell RA, Tibbs PA, Regine WF, Dempsey RJ, Mohiuddin M, Kryscio RJ, et al: Postoperative radiotherapy in the treatment of single metastases to the brain: a randomized trial. JAMA 280:1485-1489, 1998

19. Patchell RA, Tibbs PA, Walsh JW, Dempsey RJ, Maruyama Y, Kryscio RJ, et al: A randomized trial of surgery in the treatment of single metastases to the brain. N Engl J Med 322:494500,1990

20. Roberge D, Parney I, Brown PD: Radiosurgery to the postoperative surgical cavity: who needs evidence? Int J Radiat Oncol Biol Phys 83:486-493, 2012

21. Smith TR, Lall RR, Lall RR, Abecassis IJ, Arnaout OM, Marymont $\mathrm{MH}$, et al: Survival after surgery and stereotactic radiosurgery for patients with multiple intracranial metastases: results of a single-center retrospective study. Clinical article. J Neurosurg [epub ahead of print], 2014

22. Vogelbaum MA, Angelov L, Lee SY, Li L, Barnett GH, Suh JH: Local control of brain metastases by stereotactic radiosurgery in relation to dose to the tumor margin. J Neurosurg 104:907-912, 2006

Manuscript submitted July 1, 2014

Accepted July 29, 2014.

Portions of this work were presented at the 17th International Leksell Gamma Knife Society Meeting, New York, New York, May 11-15, 2014, and at the 16th Biennial Canadian Neuro-Oncology Meeting, Halifax, Nova Scotia, Canada, June 12-14, 2014.

Please include this information when citing this paper: DOI: 10.3171/2014.7.GKS141488.

Address correspondence to: David Mathieu, M.D., F.R.C.S.C., CHUS, Service de Neurochirurgie, 3001, 12e Ave. Nord, Sherbrooke, QC J1H 5N4, Canada. email: david mathieu@usherbrooke. ca. 